\title{
Chaos for Discrete Dynamical System
}

\author{
Lidong Wang, ${ }^{1,2}$ Heng Liu, ${ }^{1,2}$ and Yuelin Gao ${ }^{1}$ \\ ${ }^{1}$ Information and Computational Science department, Beifang University of Nationality, Yinchuan, Ningxia 750021, China \\ ${ }^{2}$ School of Science, Dalian Nationalities University, Dalian, Liaoning 116600, China \\ Correspondence should be addressed to Lidong Wang; wld0117@yahoo.cn
}

Received 8 January 2013; Revised 1 March 2013; Accepted 2 March 2013

Academic Editor: Ezio Venturino

Copyright (C) 2013 Lidong Wang et al. This is an open access article distributed under the Creative Commons Attribution License, which permits unrestricted use, distribution, and reproduction in any medium, provided the original work is properly cited.

We prove that a dynamical system is chaotic in the sense of Martelli and Wiggins, when it is a transitive distributively chaotic in a sequence. Then, we give a sufficient condition for the dynamical system to be chaotic in the strong sense of Li-Yorke. We also prove that a dynamical system is distributively chaotic in a sequence, when it is chaotic in the strong sense of Li-Yorke.

\section{Introduction}

Since Li and Yorke first gave the definition of chaos by using strict mathematical language in 1975 [1], the research on chaos has greatly influenced modern science, not just natural sciences but also several social sciences, such as economics, sociology, and philosophy. The theory of chaos convinced scientists that a simple definite system can produce complicated features and a complex system instead possibly follows a simple law. However, scientists in different fields, finding different chaotic connotations, gave different definitions of chaos such as Li-Yorke chaos, distributional chaos, and Devaney chaos. In order to establish a satisfactory definitional and terminological framework for complex dynamical systems that are based on strict mathematical definitions, these concepts with less ambiguous are necessary, and their interdependence has to be clarified. There is no doubt that the mathematical definition of Li-Yorke chaos has a large influence than any other one, whereas distributional chaos possesses some statistical connotations besides the uncertainty of long-term behaviors. So, comparing distributional chaos with Li-Yorke chaos is a meaningful and significant problem.

In order to reveal the inner relations between Li-Yorke chaos and distributional chaos, the author brought up the definition of distributional chaos in a sequence in [2]. In this paper, we mainly prove the relations between some different chaoses in discrete dynamical systems.

The main theorems are stated as follows.
Theorem 1. If a dynamical system $(X, f)$ exhibits transitive distributional chaos in a sequence, then,

(1) it is chaotic in the sense of Martelli;

(2) it is chaotic in the sense of Wiggins.

Theorem 2. Let $a, b \in X$ with $a \neq b$ and $p_{k} \rightarrow \infty$ be a sequence of positive integers. If for any sequence $C=C_{1} \cdots C_{k} \cdots$ where $C_{K}=\overline{B(a, 1 / k)}$ or $B(b, 1 / k)(B(a, 1 / k)=\{x \mid d(a, x)<$ $(1 / k)\})$ there exists $x(C) \in X$, such that for each $k \geq$ $1, f^{p_{k}}(x(C)) \in C_{k}$ then system $(X, f)$ is chaotic in the strong sense of Li-Yorke.

Theorem 3. If a dynamical system $(X, f)$ exhibits chaotic in the strong sense of Li-Yorke, then it is distributively chaotic in a sequence.

\section{Problem Statement and Preliminaries}

Throughout this paper $X$ will denote a compact metric space with metric $d$.

2.1. Several Definitions and Lemmas. $D \subset X$ is said to be a chaotic set of $f$ if for any pair $(x, y) \in D \times D, x \neq y$, we have

$$
\begin{aligned}
& \lim _{n \rightarrow \infty} \inf d\left(f^{n}(x), f^{n}(y)\right)=0, \\
& \lim _{n \rightarrow \infty} \sup d\left(f^{n}(x), f^{n}(y)\right)>0 .
\end{aligned}
$$


Definition 4. $f$ is said to be chaotic in the sense of Li and Yorke (for short: Li-Yorke chaotic), if it has a chaotic set $D$ which is uncountable.

Let $\left\{p_{i}\right\}$ be an increasing sequence of positive integers, $x, y \in X, t>0$. Let

$$
\begin{aligned}
& F_{x y}\left(t,\left\{p_{i}\right\}\right)=\lim _{n \rightarrow \infty} \inf \frac{1}{n} \sum_{k=1}^{n} \chi_{[0, t)}\left(d\left(f^{p_{k}}(x), f^{p_{k}}(y)\right)\right), \\
& F_{x y}^{*}\left(t,\left\{p_{i}\right\}\right)=\lim _{n \rightarrow \infty} \sup \frac{1}{n} \sum_{k=1}^{n} \chi_{[0, t)}\left(d\left(f^{p_{k}}(x), f^{p_{k}}(y)\right)\right),
\end{aligned}
$$

where $\chi_{A}(y)$ is 1 if $y \in A$ and 0 otherwise. Obviously, $F_{x y}$ and $F_{x y}^{*}$ are both nondecreasing functions. If for $t \leq 0$ we define $F_{x y}(t)=F_{x y}^{*}(t)=0$, then $F_{x y}$ and $F_{x y}^{*}$ are probability distributional functions.

Definition 5. Let $D \subset X$, If $\forall x, y \in D, x \neq y$, we have

$$
\begin{aligned}
& \text { (1) } \exists \delta>0, \quad F_{x y}=\left(\delta,\left\{p_{i}\right\}\right)=0, \\
& \text { (2) } \forall t>0, \quad F_{x y}^{*}\left(t,\left\{p_{i}\right\}\right)=1,
\end{aligned}
$$

then $D$ is said to be a distributively chaotic set in a sequence. The two points are said to be distributively chaotic point pair in a sequence. $f$ is said to be distributively chaotic in a sequence, if $f$ has a distributively chaotic set in a sequence which is uncountable.

Definition 6. Let $S \subset X$. If there exist two strictly increasing sequences of positive integers $\left\{p_{i}\right\}$ and $\left\{q_{i}\right\}$ such that for any $x, y \in S, x \neq y$,

$$
\begin{aligned}
& \lim _{i \rightarrow \infty} d\left(f^{p_{i}}(x), f^{p_{i}}(y)\right)=0, \\
& \lim _{i \rightarrow \infty} d\left(f^{q_{i}}(x), f^{q_{i}}(y)\right)>0,
\end{aligned}
$$

then $S$ is said to be a strong scrambled set. $f$ is said to be chaotic in the strong sense of Li-Yorke, if $f$ has an uncountable strong scrambled set.

Definition 7 . Let $\left\{p_{i}\right\}$ be an increasing sequence of positive integers, then,

$$
\begin{aligned}
& \operatorname{PR}\left(f,\left\{p_{i}\right\}\right) \\
& =\{(x, y) \in X \times X \mid \forall \varepsilon>0, \\
& \left.\quad \exists i \in N \text { such that } d\left(f^{p_{i}}(x), f^{p_{i}}(y)\right)<\varepsilon\right\}
\end{aligned}
$$

is called proximal relation with respect to $\left\{p_{i}\right\}$.

Thus

$$
\begin{aligned}
& \operatorname{AR}\left(f,\left\{p_{i}\right\}\right) \\
& =\left\{(x, y) \in X \times X \mid \lim _{i \rightarrow \infty} d\left(f^{p_{i}}(x), f^{p_{i}}(y)\right)=0\right\}
\end{aligned}
$$

is called asymptotic relation with respect to $\left\{p_{i}\right\}$.
Thus

$$
\operatorname{DR}\left(f,\left\{p_{i}\right\}\right)=X \times X-\operatorname{PR}\left(f,\left\{p_{i}\right\}\right)
$$

is called distal relation with respect to $\left\{p_{i}\right\}$.

So

$$
\begin{aligned}
& \operatorname{DCR}\left(f,\left\{p_{i}\right\}\right) \\
& =\{(x, y) \in X \times X \mid(x, y) \\
& \left.\quad \text { is a distributively chaotic point pair of } f \text { in } p_{i}\right\}
\end{aligned}
$$

is called distributively chaotic respect to $\left\{p_{i}\right\}$.

Definition 8 (see [3]). $f$ is (topologically) transitive if for any two nonempty open sets $U, V \subset X$ there exists $n>0$ such that $f^{n}(U) \cap V \neq \emptyset$. $f$ is (topologically) weakly mixing if for any three nonempty open sets $U, V, W \subset X$ there exists $n>0$ such that $f^{n}(W) \cap V \neq \emptyset$ and $f^{n}(W) \cap U \neq \emptyset$.

Definition 9 (see [4-6]). Let $f$ be a continuous map from a compact metric space $(X, d)$ into itself. The orbit of a point $x \in X$ is said to be unstable if there exists $r>0$ such that for every $\epsilon>0$ there are $y \in X$ and $n \geq 1$ satisfying inequalities $d(x, y)<\epsilon$ and $d\left(f^{n}(x), f^{n}(y)\right)>r$. The map $f$ is said to be chaotic in the sense of Martelli if there exists $x_{0} \in X$ such that $x_{0}$ has dense orbit which is unstable.

Definition 10 (see [7]). Let $f$ be a continuous map from a compact metric space $(X, d)$ into itself. We say $f$ has sensitive dependence on initial conditions if there exists $r>0$ such that for any $x \in X$ and $\epsilon>0$, there is some $y \in X$ and a nonnegative integer $n$ satisfying $d(x, y)<\epsilon$ and $d\left(f^{n}(x), f^{n}(y)\right)>$ $r$. $f$ is said to be chaotic in the sense of Wiggins, if $f$ is transitive and has sensitive dependence on initial conditions.

Definition 11. Let $(X, d)$ be a compact metric space, $f$ : $X \rightarrow X$ be a continuous map, and $D$ be an uncountable distributively scrambled set in a sequence.

We say that $f$ exhibits dense distributional chaos in a sequence if the set $D$ may be chosen to be dense. If $D$ is not only dense but additionally consists of points with dense orbits, then we say that $f$ exhibits transitive distributional chaos in a sequence.

Lemma 12. Let $\Sigma$ be an infinite sequence set of $\{0,1\}$. Then, there exists an uncountable subset $E \subset \sum$ such that for any different points $s=s_{1} s_{2}, \ldots, t=t_{1} t_{2}, \ldots, s_{m} \neq t_{m}$ for infinitely many $m$ and $s_{n}=t_{n}$ for infinitely many $n$.

Proof. For a proof, see [8].

Lemma 13. If $\left\{p_{i}\right\}$ and $\left\{q_{i}\right\}$ are both infinite increasing subsequences of $\left\{m_{i}\right\}$ which is a sequence of positive integers, then there exists an infinite increasing subsequence $\left\{t_{i}\right\} \subset\left\{m_{i}\right\}$ such that

$$
\operatorname{AR}\left(f,\left\{p_{i}\right\}\right) \cap \operatorname{DR}\left(f,\left\{q_{i}\right\}\right) \subset \operatorname{DCR}\left(f,\left\{t_{i}\right\}\right) .
$$


Proof. For a proof, see [9].

Lemma 14. $f$ is weakly mixing if and only if for any $m \geq 2, f_{m}$ is transitive.

Proof. For a proof, see [10].

\section{Proof of Main Theorem}

Proof of Theorem 1. (1) There is no isolated points in $X$ as otherwise the set of points with dense orbit is at most countable. But in the case of compact set without isolated points, the existence of dense orbit implies transitivity.

Let $D$ be a dense scrambled set in the sequence $\left\{p_{k}\right\}$ consisting of transitive points, and let $r>0$ be such that $F_{x y}\left(r,\left\{p_{k}\right\}\right)=0$ for all distinct $x, y \in D$. Let us fix any $x_{0} \in D$. Because orbit of $x_{0}$ is dense, for any $\epsilon>0$, there exists $y \in D$ and $k \geq 1$ satisfying the inequalities $d\left(x_{0}, y\right)<\epsilon$ and $F_{x_{0} y}\left(r,\left\{p_{k}\right\}\right)=0$. This implies that $d\left(f^{p_{k}}\left(x_{0}\right), f^{p_{k}}(y)\right)>r$ for some $k \geq 1$. This shows that the orbit of $x_{0}$ is unstable. So, $(X, f)$ is chaotic in the sense of Martelli.

(2) Fix any $\epsilon>0$. In $\epsilon$-neighborhood of any point $x$, we can find points $y, z \in D$ such that $d\left(f^{p_{k}}(y), f^{p_{k}}(z)\right)>r$. Then, $d\left(f^{p_{k}}(x), f^{p_{k}}(y)\right)>r / 2$ or $d\left(f^{p_{k}}(x), f^{p_{k}}(z)\right)>r / 2$.

Proof of Theorem 2. Let $E$ be an uncountable subset of $\Sigma$, as in Lemma 12. For each $s=s_{0} s_{1} \cdots s_{n} \cdots \in E$, by the hypotheses, we can choose a point $x(s) \in X$ such that for any $k$, if $n !<$ $k \leq(n+1)$ ! then,

$$
x_{p_{k}}^{s} \in \begin{cases}\overline{B\left(a, \frac{1}{k}\right)} & \text { if } s_{n}=0, \\ \overline{B\left(b, \frac{1}{k}\right)} & \text { if } s_{n}=1 .\end{cases}
$$

Put $D=\left\{x^{s} \mid s \in E\right\}$. Clearly, if $s \neq t$ then $x^{s} \neq x^{t}$. It follows that $E$ being uncountable implies so is $D$.

Let $x^{s}, x^{t} \in D$ be any different points, where $s=s_{0} s_{1}$ $\cdots s_{i} \cdots, t=t_{0} t_{1} \cdots t_{i} \cdots \in E$. By the property of $E$, we know that there exist sequences of positive integers $m_{i} \rightarrow$ $\infty, n_{i} \rightarrow \infty$ such that $s_{m_{i}} \neq t_{m_{i}}, s_{n_{i}}=t_{n_{i}}$ for all $i$, and for $m_{i}$ large enough $1 / m<d(a, b) / 4=\delta$, we have $d\left(x_{m_{i}}^{s}, x_{m_{i}}^{t}\right)>\delta$. Thus,

$$
\lim _{i \rightarrow \infty} d\left(x_{m_{i}}^{s}, x_{m_{i}}^{t}\right) \geq \delta
$$

this shows

$$
\lim _{i \rightarrow \infty} \sup d\left(x_{m_{i}}^{s}, x_{m_{i}}^{t}\right) \geq \delta
$$

Meanwhile, for $n_{i}$ large enough, $x_{n_{i}}^{s}$ and $x_{n_{i}}^{t}$ lie in the same ball of diameter less than $1 / n_{i}$. Thus, $d\left(x_{n_{i}}^{s}, x_{n_{i}}^{t}\right)<1 / n$, so

$$
\lim _{i \rightarrow \infty} d\left(x_{n_{i}}^{s}, x_{n_{i}}^{t}\right)=0 \text {. }
$$

This shows

$$
\lim _{i \rightarrow \infty} \inf d\left(x_{n_{i}}^{s}, x_{n_{i}}^{t}\right)=0 .
$$

Above all, $(X, f)$ is chaotic in the strong sense of Li-Yorke.
Proof of Theorem 3. Because $f$ is chaotic in the strong sense of Li-Yorke, there exists an infinite increasing sequence $\left\{p_{i}\right\} \subset \mathbb{N}$ and uncountable set $S \subset X$, such that for any $x, y \in S$ with $x \neq y$, we have

$$
\lim _{i \rightarrow \infty} d\left(f^{p_{i}}(x), f^{p_{i}}(y)\right)=0
$$

so that $(x, y) \in \operatorname{AR}\left(f,\left\{p_{i}\right\}\right)$.

Again, by the definition of chaos in the strong sense of Li-Yorke, there exists $\left\{q_{i}\right\} \subset \mathbb{N}$, such that

$$
\lim _{i \rightarrow \infty} d\left(f^{q_{i}}(x), f^{q_{i}}(y)\right)>0
$$

so that $(x, y) \in \operatorname{DR}\left(f,\left\{q_{i}\right\}\right)$.

Hence, $S \times S-\Delta \subset \operatorname{AR}\left(f,\left\{p_{i}\right\}\right) \cap \operatorname{DR}\left(f,\left\{q_{i}\right\}\right)$, where $\Delta=\{(x, x) ; x \in X\}$. Then by Lemma 13, there exists a subsequence $\left\{t_{i}\right\} \subset \mathbb{N}$ such that $S \times S \subset \operatorname{DCR}\left(f,\left\{t_{i}\right\}\right)$. This shows that $S$ is a distributively chaotic set in the sequence $\left\{t_{i}\right\}$ of $f$.

Corollary 15. If system $(X, f)$ satisfies conditions of Theorem 2. then it is distributively chaotic in the sequence.

Proof. By Theorems 2 and 3, we can easily prove it.

Corollary 16. Let $(X, d)$ be a locally compact metric space containing at least two points. If system $(X, f)$ is weakly mixing, then it must be chaotic in the strong sense of Li-Yorke.

Proof. Let $f$ be weakly mixing, $a, b \in X$ with $a \neq b$. Take arbitrarily a nonempty open set $V_{0} \subset X$ such that $\bar{V}_{0}$ is compact. Since $f$ is weakly mixing, there exists $p_{1}>0$ such that $f^{p_{1}}\left(V_{0}\right) \cap B(a, 1 / k) \neq \emptyset$ and $f^{p_{1}}\left(V_{0}\right) \cap B(b, 1 / k) \neq \emptyset$. Thus, we find points $x_{1}, x_{2}$ such that $f^{p_{1}}\left(x_{1}\right) \in B(a, 1 / k), f^{p_{1}}\left(x_{2}\right) \in$ $B(b, 1 / k)$. Assume that there exist positive integers $p_{1}<p_{2}<$ $\cdots<p_{k}$ such that for each finite sequence $A_{1} A_{2} \cdots A_{k}$, where $A_{i} \in\{B(a, 1 / i), B(b, 1 / i)\}$, there is a point $x \in V_{0}$ satisfying $f^{p_{i}}(x) \in A_{i}$ for $i=1,2, \ldots, k$, the set of all such points will denoted by $S_{k}$. By continuity of $f$, each $x \in S_{k}$ has an open nonempty neighborhood $W_{x} \subset V_{0}$ such that $f^{p_{i}}\left(W_{x}\right) \subset A_{i}$, if $f^{p_{i}}(x) \in A_{i}$, it follows from Lemma 14 that there exists $p_{k+1}>$ $p_{k}$ such that for each $x \in S_{k}, f^{p_{k+1}}\left(W_{x}\right) \cap B(a, 1 /(k+1)) \neq \emptyset$ and $f^{p_{k+1}}\left(W_{x}\right) \cap B(b, 1 /(k+1)) \neq \emptyset$. Thus by induction, we know that there exists a sequence $p_{k} \rightarrow \infty$ of positive integers such that for any finite sequence $A_{1} \cdots A_{k}$, there is a point $x \in V_{0}$ satisfying $f^{p_{i}}(x) \in A_{i}, 1 \leq i \leq k$.

Let $C=C_{1} C_{2} \cdots$ be an infinite sequence, where

$$
C_{k} \in\left\{\overline{B\left(a, \frac{1}{k}\right)}, \overline{B\left(b, \frac{1}{k}\right)}\right\} .
$$

For each $k$, we can take a point $x_{k} \in C_{k}$ such that

$$
f^{p_{i}}\left(x_{k}\right) \in C_{i}, \quad 1 \leq i \leq k .
$$

Since $\bar{V}_{0}$ is compact, the infinite sequence $\left\{x_{i}\right\}$ has a limit point in $\bar{V}_{0}$, say $x_{C}$, it is not difficult to show $f^{p_{k}}\left(x_{C}\right) \subset C_{k}$.

Thus by Theorem 2, $f$ is a strong chaos in the sense of LiYorke. 


\section{Acknowledgment}

This work is supported by the NSFC no. 11271061, the NSFC no. 11001038, the NSFC no. 61153001, and the Independent Research Foundation of the Central Universities no. DC 12010111.

\section{References}

[1] T. Y. Li and J. A. Yorke, "Period three implies chaos", The American Mathematical Monthly, vol. 82, no. 10, pp. 985-992, 1975.

[2] L. D. Wang, G. F. Huang, and S. M. Huan, "Distributional chaos in a sequence," Nonlinear Analysis, vol. 67, no. 7, pp. 2131-2136, 2007.

[3] P. Oprocha, "Distributional chaos revisited," Transactions of the American Mathematical Society, vol. 361, no. 9, pp. 4901-4925, 2009.

[4] M. Martelli, Introduction to Discrete Dynamical Systems and Chaos, Discrete Mathematics and Optimization, John Wiley \& Sons, New York, NY, USA, 1999.

[5] M. Martelli, M. Dang, and T. Seph, "Defining chaos," Mathematics Magazine, vol. 71, no. 2, pp. 112-122, 1998.

[6] L. Liu and S. Zhao, "Martelli’s Chaos in inverse limit dynamical systems and hyperspace dynamical systems," Results in Mathematics, vol. 63, no. 1-2, pp. 195-207, 2013.

[7] S. Wiggins, Introduction to Applied Nonlinear Dynamical Systems and Chaos, vol. 2, Springer, New York, NY, USA, 1990.

[8] L. Wang, G. Liao, and Y. Yang, "Recurrent point set of the shift on $\Sigma$ and strong chaos," Annales Polonici Mathematici, vol. 78, no. 2, pp. 123-130, 2002.

[9] R. S. Yang, "Distribution chaos in a sequence and topologically mixing," Acta Mathematica Sinica, vol. 45, no. 4, pp. 752-758, 2002 (Chinese).

[10] G. Liao, L. Wang, and Y. Zhang, "Transitivity, mixing and chaos for a class of set-valued mappings," Science in China A, vol. 49, no. 1, pp. 1-8, 2006. 


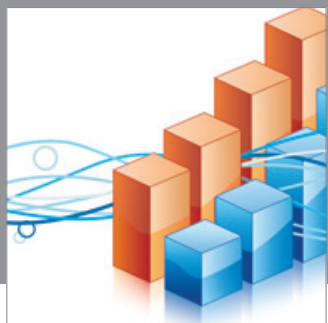

Advances in

Operations Research

mansans

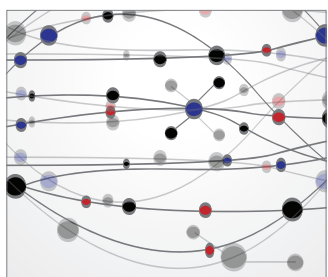

The Scientific World Journal
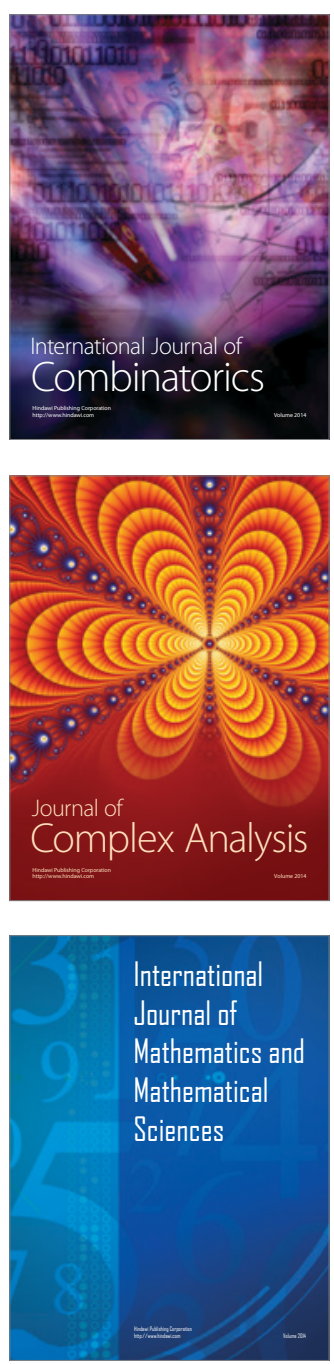
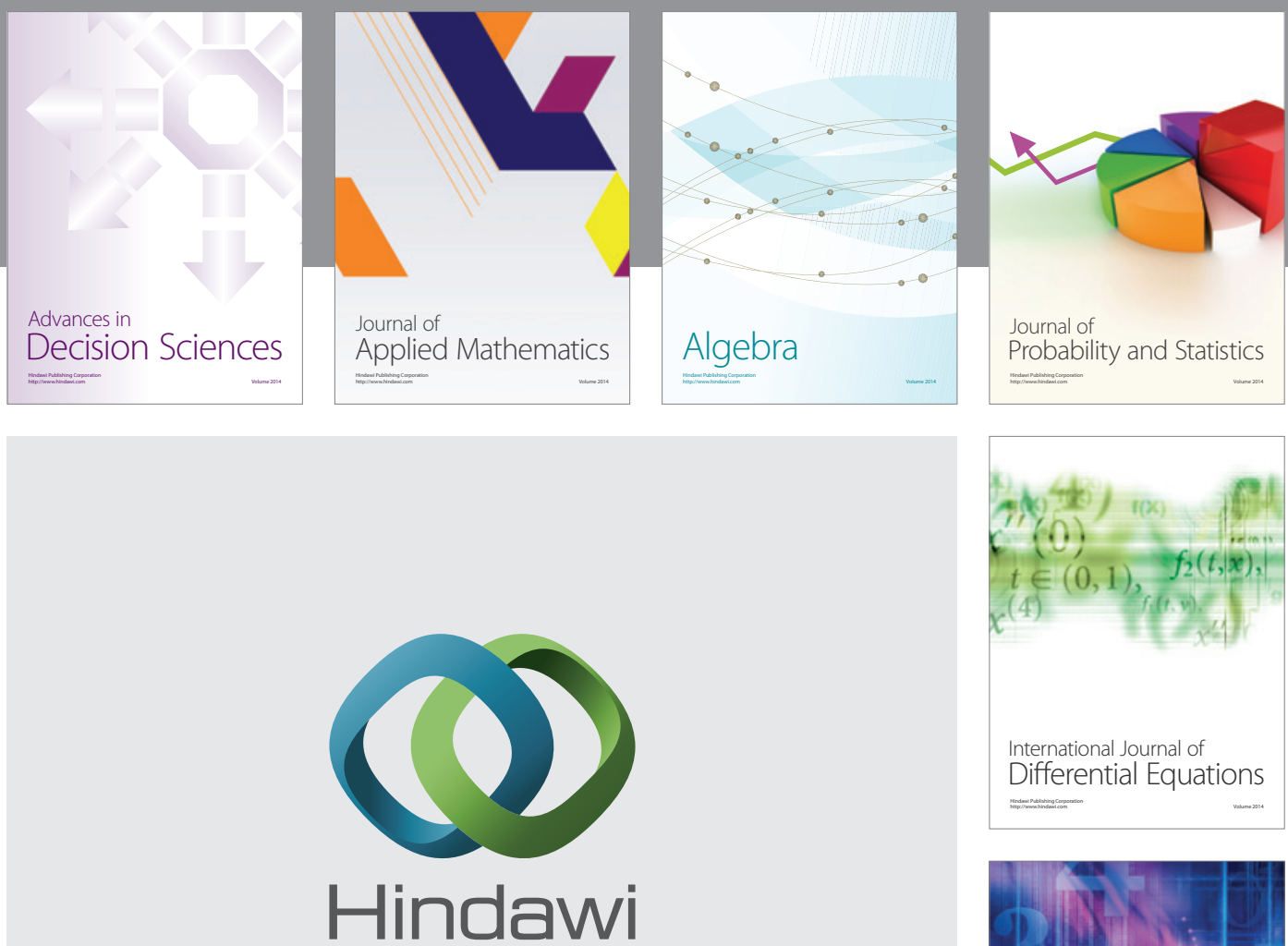

Submit your manuscripts at http://www.hindawi.com
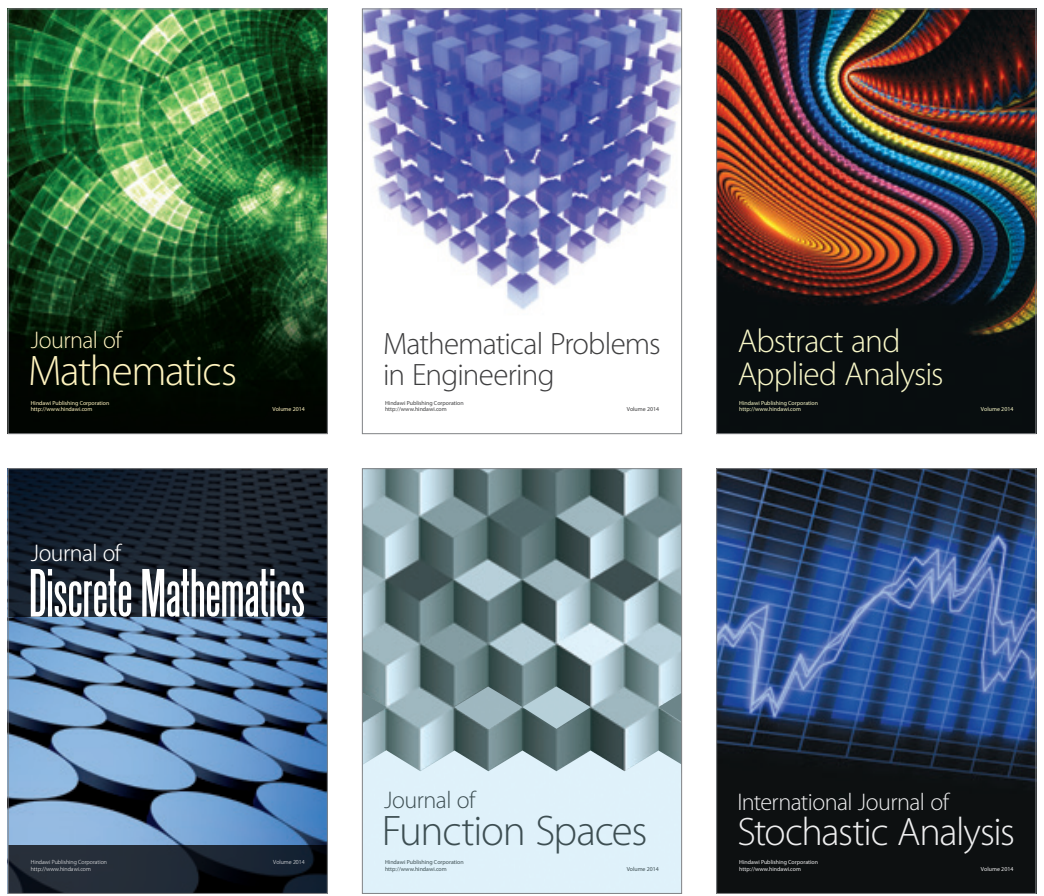

Journal of

Function Spaces

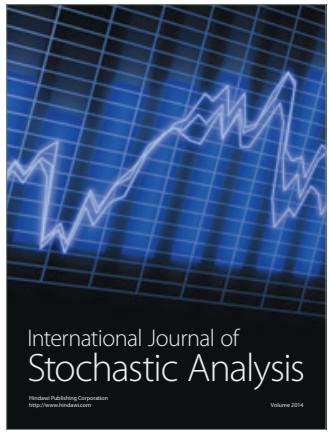

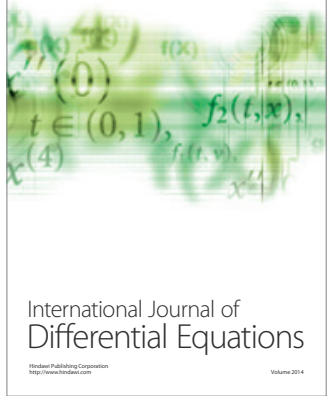
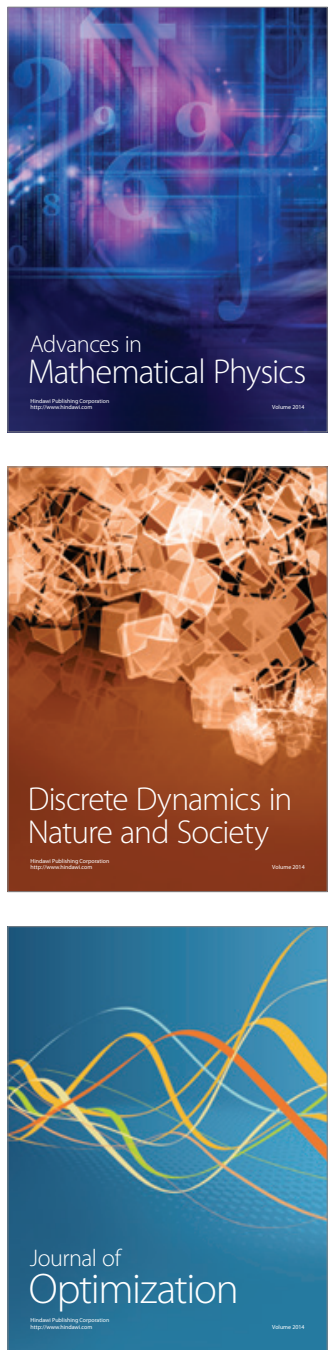